International Journal of Pure and Applied Mathematics

Volume 87 No. 5 2013, 699-706

ISSN: 1311-8080 (printed version); ISSN: 1314-3395 (on-line version)

url: http://www.ijpam.eu

doi: http://dx.doi.org/10.12732/ijpam.v87i5.3

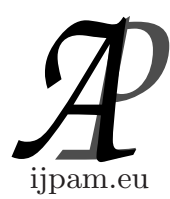

\title{
FINSLERIAN HYPERSURFACES AND RANDERS CONFORMAL CHANGE OF A FINSLER METRIC
}

\author{
H.S. Shukla ${ }^{1}$, V.K. Chaubey ${ }^{2}$, Arunima Mishra ${ }^{3} \S$ \\ ${ }^{1,3}$ Department of Mathematics and Statistics \\ D.D.U. Gorakhpur University \\ Gorakhpur U.P., 273009, INDIA \\ ${ }^{2}$ Department of Applied Sciences \\ Ansal Technical Campus, Sector-C \\ Pocket-9, Sushant Golf City, Lucknow U.P., 226030, INDIA
}

\begin{abstract}
In the present paper we have studied the Finslerian hypersurfaces and Randers conformal change of a Finsler metric. The relations between the Finslerian hypersurface and the other which is Finslerian hypersurface given by Randers conformal change have been obtained. We have also proved that Randers conformal change makes three types of hypersurfaces invariant under certain condition. These three types of hypersurfaces are hyperplanes of first, second and third kind.
\end{abstract}

AMS Subject Classification: 53B40, 53C60

Key Words: Finslerian hypersurfaces, randers conformal change, randers change, conformal change, hyperplanes of first, second and third kind

\section{Introduction}

Let $\left(M^{n}, L\right)$ be an n-dimensional Finsler space on a differentiable manifold $M^{n}$, equipped with the fundamental function $L(x, y)$. In 1984, Shibata [3] introduced

Received: $\quad$ March 17, 2013

(C) 2013 Academic Publications, Ltd.

${ }^{\S}$ Correspondence author url: www.acadpubl.eu 
the transformation of Finsler metric:

$$
L^{\prime}(x, y)=f(L, \beta)
$$

where $\beta=b_{i}(x) y^{i}, \quad b_{i}(x)$ are components of a covariant vector in $\left(M^{n}, L\right)$ and $f$ is positively homogeneous function of degree one in $L$ and $\beta$. This change of metric is called a $\beta$-change.

The conformal theory of Finsler spaces has been initiated by M.S. Knebelman [4] in 1929 and has been investigated in detail by many authors [5, 6, 7, 8] etc. The conformal change is defined as

$$
L(x, y) \rightarrow e^{\sigma(x)} L(x, y),
$$

where $\sigma(x)$ is a function of position only and known as conformal factor.

In the year 2012 [10] we studied Randers conformal change defining as

$$
L(x, y) \rightarrow L^{*}(x, y)=e^{\sigma(x)} L(x, y)+\beta(x, y),
$$

where $\sigma(x)$ is a function of $\mathrm{x}$ and $\beta(x, y)=b_{i}(x) y^{i}$ is a 1 - form on $M^{n}$. This change generalizes various types of changes. When $\beta=0$, it reduces to a conformal change. When $\sigma=0$, it reduces to a Randers change. When $\beta=0$ and $\sigma$ is a non-zero constant then it reduces to homothetic change.

On the other hand, in $1985 \mathrm{M}$. Matsumoto investigated the theory of Finslerian hypersurface [2]. He has defined three types of hypersurfaces that were called a hyperplane of the first, second and third kinds.

In the year 2005, Prasad and Tripathi [12] studied the Finslerian Hypersurfaces and Kropina change of a Finsler metric and obtained different results in his paper. In the present paper, using the field of linear frame $[1,9,11]$ we shall consider Finslerian hypersurfaces given by a Randers conformal change of a Finsler metric. Our purpose is to give some relations between the original Finslerian hypersurface and the other which is Finselrian hypersurface given by Randers conformal change. We also obtain that a Randers conformal change makes three types of hypersurfaces invariant under certain condition.

\section{Finslerian Hypersurfaces}

Let $M^{n}$ be an n-dimensional smooth manifold and $F^{n}=\left(M^{n}, L\right)$ be an ndimensional Finsler space equipped with the fundamental function $L(x, y)$ on $M^{n}$. The metric tensor $g_{i j}(x, y)$ and Cartan's C-tensor $C_{i j k}(x, y)$ are given by

$$
g_{i j}=\frac{1}{2} \frac{\partial^{2} L^{2}}{\partial y^{i} \partial y^{j}}, \quad C_{i j k}=\frac{1}{2} \frac{\partial g_{i j}}{\partial y^{k}}
$$


respectively and we introduce the Cartan's connection $C \Gamma=\left(F_{j k}^{i}, N_{j}^{i}, C_{j k}^{i}\right)$ in $F^{n}$.

A hypersurface $M^{n-1}$ of the underlying smooth manifold $M^{n}$ may be parametrically represented by the equation $x^{i}=x^{i}\left(u^{\alpha}\right)$, where $u^{\alpha}$ are Gaussian coordinates on $M^{n-1}$ and Greek indices vary from 1 to n-1. Here we shall assume that the matrix consisting of the projection factors $B_{\alpha}^{i}=\frac{\partial x^{i}}{\partial u^{\alpha}}$ is of rank $\mathrm{n}-1$. The following notations are also employed:

$$
B_{\alpha \beta}^{i}=\frac{\partial^{2} x^{i}}{\partial u^{\alpha} \partial u^{\beta}}, \quad B_{0 \beta}^{i}=v^{\alpha} B_{\alpha \beta}^{i}
$$

If the supporting element $y^{i}$ at a point $\left(u^{\alpha}\right)$ of $M^{n-1}$ is assumed to be tangential to $M^{n-1}$, we may then write $y^{i}=B_{\alpha}^{i}(u) v^{\alpha}$ i.e. $v^{\alpha}$ is thought of as the supporting element of $M^{n-1}$ at the point $\left(u^{\alpha}\right)$. Since the function $\bar{L}(u, v)=L\{x(u), y(u, v)\}$ gives rise to a Finsler metric of $M^{n-1}$, we get a $(n-1)$-dimensional Finsler space $F^{n-1}=\left\{M^{n-1}, \bar{L}(u, v)\right\}$.

At each point $\left(u^{\alpha}\right)$ of $F^{n-1}$, the unit normal vector $N^{i}(u, v)$ is defined by

$$
g_{i j} B_{\alpha}^{i} N^{j}=0, \quad g_{i j} N^{i} N^{j}=1
$$

If $B_{i}^{\alpha}, N_{i}$ is the inverse matrix of $\left(B_{\alpha}^{i}, N^{i}\right)$, we have

$$
B_{\alpha}^{i} B_{i}^{\beta}=\delta_{\alpha}^{\beta}, \quad B_{\alpha}^{i} N_{i}=0, \quad N^{i} N_{i}=1 \quad \text { and } \quad B_{\alpha}^{i} B_{j}^{\alpha}+N^{i} N_{j}=\delta_{j}^{i} .
$$

Making use of the inverse matrix $\left(g^{\alpha \beta}\right)$ of $\left(g_{\alpha \beta}\right)$, we get

$$
B_{i}^{\alpha}=g^{\alpha \beta} g_{i j} B_{\beta}^{j}, \quad N_{i}=g_{i j} N^{j}
$$

For the induced Cartan's connection $I C \Gamma=\left(F_{\beta \gamma}^{\alpha}, N_{\alpha}^{\beta}, C_{\beta \gamma}^{\alpha}\right)$ on $F^{n-1}$, the second fundamental $h$-tensor $H_{\alpha \beta}$ and the normal curvature vector $H_{\alpha}$ are respectively given by $[2]$

$$
H_{\alpha \beta}=N_{i}\left(B_{\alpha \beta}^{i}+F_{j k}^{i} B_{\alpha}^{j} B_{\beta}^{k}\right)+M_{\alpha} H_{\beta}, \quad H_{\alpha}=N_{i}\left(B_{0 \beta}^{i}+N_{j}^{i} B_{\beta}^{j}\right)
$$

where

$$
M_{\alpha}=C_{i j k} B_{\alpha}^{i} N^{j} N^{k}
$$

Contracting $H_{\alpha \beta}$ by $v^{\alpha}$, we immediately get $H_{0 \beta}=H_{\alpha \beta} v^{\alpha}=H_{\beta}$. Furthermore the second fundamental $v$-tensor $M_{\alpha \beta}$ is given by [2]

$$
M_{\alpha \beta}=C_{i j k} B_{\alpha}^{i} B_{\beta}^{j} N^{k}
$$




\section{Finsler Space with Randers Conformal Change}

Let $\left(M^{n}, \mathrm{~L}\right)$ be a Finsler space $F^{n}$, where $M^{n}$ is an n-dimensional differentiable manifold equipped with a fundamental function L. A change in fundamental metric L, defined by equation (2), is called Randers conformal change, where $\sigma(x)$ is conformal factor and function of position only and $\beta(x, y)=b_{i}(x) y^{i}$ is a 1- form on $M^{n}$. A space equipped with fundamental metric $L^{*}(x, y)$ is called Randers conformally changed space $F^{* n}$.

Differentiating equation (2) with respect to $y^{i}$, the normalized supporting element $l_{i}^{*}=\dot{\partial}_{i} L^{*}$ is given by

$$
l_{i}^{*}(x, y)=e^{\sigma(x)} l_{i}(x, y)+b_{i}(x),
$$

where $l_{i}=\dot{\partial}_{i} L$ is the normalized supporting element in the Finsler space $F^{n}$.

Differentiating (7) with respect to $y^{j}$, the angular metric tensor $h_{i j}^{*}=$ $L^{*} \dot{\partial}_{i} \dot{\partial}_{j} L^{*}$ is given by

$$
h_{i j}^{*}=e^{\sigma(x)} \frac{L^{*}}{L} h_{i j}
$$

where $h_{i j}=L \dot{\partial}_{i} \dot{\partial}_{j} L$ is the angular metric tensor in the Finsler space $F^{n}$.

Again the fundamental tensor $g_{i j}^{*}=\dot{\partial}_{i} \dot{\partial}_{j} \frac{L^{* 2}}{2}=h_{i j}^{*}+l_{i}^{*} l_{j}^{*}$ is given by

$$
g_{i j}^{*}=\tau g_{i j}+b_{i} b_{j}+e^{\sigma(x)} L^{-1}\left(b_{i} y_{j}+b_{j} y_{i}\right)-\beta e^{\sigma(x)} L^{-3} y_{i} y_{j}
$$

where we put $y_{i}=g_{i j}(x, y) y^{j}, \tau=e^{\sigma(x)} \frac{L^{*}}{L}$ and $g_{i j}$ is the fundamental tensor of the Finsler space $F^{n}$. It is easy to see that the $\operatorname{det}\left(g_{i j}^{*}\right) \operatorname{does}$ not vanish, and the reciprocal tensor with components $g^{* i j}$ is given by

$$
g^{* i j}=\tau^{-1} g^{i j}+\phi y^{i} y^{j}-L^{-1} \tau^{-2}\left(y^{i} b^{j}+y^{j} b^{i}\right)
$$

where $\phi=e^{-2 \sigma(x)}\left(L e^{\sigma(x)} b^{2}+\beta\right) L^{*-3}, b^{2}=b_{i} b^{i}, b^{i}=g^{i j} b_{j}$ and $g^{i j}$ is the reciprocal tensor of $g_{i j}$.

Here it will be more convenient to use the tensors

$$
h_{i j}=g_{i j}-L^{-2} y_{i} y_{j}, \quad a_{i}=\beta L^{-2} y_{i}-b_{i}
$$

both of which have the following interesting property:

$$
h_{i j} y^{j}=0, \quad a_{i} y^{i}=0
$$

Now differentiating equation (9) with respect to $y^{k}$ and using relation (11), the Cartan covariant tensor $C^{*}$ with the components $C_{i j k}^{*}=\dot{\partial}_{k}\left(\frac{g_{i j}^{*}}{2}\right)$ is given as:

$$
C_{i j k}^{*}=\tau\left[C_{i j k}-\frac{1}{2 L^{*}}\left(h_{i j} a_{k}+h_{j k} a_{i}+h_{k i} a_{j}\right)\right]
$$


where $C_{i j k}$ is (h)hv-torsion tensor of Cartan's connection $C \Gamma$ of Finsler space $F^{n}$.

In order to obtain the tensor with the components $C_{i k}^{* j}$, paying attention to (12), we obtain from (10) and (13),

$$
\begin{array}{r}
C_{i k}^{* j}=C_{i k}^{j}-\frac{1}{2 L^{*}}\left(h_{i}^{j} a_{k}+h_{k}^{j} a_{i}+h_{i k} a^{j}\right)-(\tau L)^{-1} C_{i k r} y^{j} b^{r}- \\
\frac{\tau^{-1}}{2 L L^{*}}\left(2 a_{i} a_{k}+a^{2} h_{i k}\right) y^{j}
\end{array}
$$

where $a_{i} a^{i}=a^{2}$.

\section{Hypersurfaces Given by a Randers Conformal Change}

Consider a Finslerian hypersurface $F^{n-1}=\left\{M^{n-1}, \bar{L}(u, v)\right\}$ of the $F^{n}$ and another Finslerian hypersurface $F^{* n-1}=\left\{M^{n-1}, \bar{L}^{*}(u, v)\right\}$ of the $F^{* n}$ given by the Randers conformal change. Let $N^{i}$ be the unit vector at each point of $F^{n-1}$ and $\left(B_{i}^{\alpha}, N_{i}\right)$ be the inverse matrix of $\left(B_{\alpha}^{i}, N^{i}\right)$. The function $B_{\alpha}^{i}$ may be considered as components of $(n-1)$ linearly independent tangent vectors of $F^{n-1}$ and they are invariant under Randers conformal change. Thus we shall show that a unit normal vector $N^{* i}(u, v)$ of $F^{* n-1}$ is uniquely determined by

$$
g_{i j}^{*} B_{\alpha}^{i} N^{* j}=0, \quad g_{i j}^{*} N^{* i} N^{* j}=1
$$

Contracting (9) by $N^{i} N^{j}$ and paying attention to (3) and the fact that $l_{i} N^{i}=0$, we have

$$
g_{i j}^{*} N^{i} N^{j}=\tau+\left(b_{i} N^{i}\right)^{2}
$$

Therefore we obtain

$$
g_{i j}^{*}\left\{ \pm \frac{N^{i}}{\sqrt{\tau+\left(b_{i} N^{i}\right)^{2}}}\right\}\left\{ \pm \frac{N^{j}}{\sqrt{\tau+\left(b_{i} N^{i}\right)^{2}}}\right\}=1
$$

Hence we can put

$$
N^{* i}=\frac{N^{i}}{\sqrt{\tau+\left(b_{i} N^{i}\right)^{2}}}
$$

where we have chosen the positive sign in order to fix an orientation.

Using equation (9), (17) and from first condition of (15) we have

$$
\left(b_{i} B_{\alpha}^{i}+e^{\sigma(x)} l_{i} B_{\alpha}^{i}\right) \frac{b_{j} N^{j}}{\sqrt{\tau+\left(b_{i} N^{i}\right)^{2}}}=0
$$


If $b_{i} B_{\alpha}^{i}+e^{\sigma(x)} l_{i} B_{\alpha}^{i}=0$, then contracting it by $v^{\alpha}$ and using $y^{i}=B_{\alpha}^{i} v^{\alpha}$ we get $\beta+e^{\sigma(x)} L=L^{*}=0$ which is contradiction to the assumption that $L^{*}>0$. Hence $b_{i} N^{i}=0$. Therefore equation (17) can be written as

$$
N^{* i}=\frac{1}{\sqrt{\tau}} N^{i}
$$

Summarizing the above we have

Proposition 4.1. If $\left\{\left(B_{\alpha}^{i}, N^{i}\right), \alpha=1,2, \ldots(n-1)\right\}$ be the filed of linear frame of the Finsler space $F^{n}$, there exist a field of linear frame $\left\{\left(B_{\alpha}^{i}, N^{* i}=\right.\right.$ $\left.\left.\frac{1}{\sqrt{\tau}} N^{i}\right), \alpha=1,2, \ldots(n-1)\right\}$ of the Finsler space $F^{* n}$ such that (15) is satisfied along $F^{* n-1}$ and then $b_{i}$ is tangential to both the hypersurfaces $F^{n-1}$ and $F^{* n-1}$.

The quantities $B_{i}^{* \alpha}$ are uniquely defined along $F^{* n-1}$ by

$$
B_{i}^{* \alpha}=g^{* \alpha \beta} g_{i j}^{*} B_{\beta}^{j}
$$

where $g^{* \alpha \beta}$ is the inverse matrix of $g_{\alpha \beta}^{*}$. Let $\left(B_{i}^{* \alpha}, N_{i}^{*}\right)$ be the inverse matrix of $\left(B_{\alpha}^{i}, N^{* i}\right)$, then we have

$$
B_{\alpha}^{i} B_{i}^{* \beta}=\delta_{\alpha}^{\beta}, \quad B_{\alpha}^{i} N_{i}^{*}=0, \quad N^{* i} N_{i}^{*}=1
$$

Furthermore $B_{\alpha}^{i} B_{j}^{* \alpha}+N^{* i} N_{j}^{*}=\delta_{j}^{i}$. We also get $N_{i}^{*}=g_{i j}^{*} N^{* j}$ which in view of (7), (9) and (19) gives

$$
N_{i}^{*}=\sqrt{\tau} N_{i}
$$

We denote the Cartan's connection of $F^{n}$ and $F^{* n}$ by $\left(F_{j k}^{i}, N_{j}^{i}, C_{j k}^{i}\right)$ and $\left(F_{j k}^{* i}\right.$, $\left.N_{j}^{* i}, C_{j k}^{* i}\right)$ respectively and put $D_{j k}^{i}=F_{j k}^{* i}-F_{j k}^{i}$ which will be called difference tensor. We choose the vector field $b_{i}$ in $F^{n}$ such that

$$
D_{j k}^{i}=A_{j k} b^{i}-B_{j k} l^{i}
$$

where $A_{j k}$ and $B_{j k}$ are components of symmetric covariant tensors of second order. Since $b_{i} N^{i}=0$ and $N_{i} l^{i}=0$, from (21) we get $N_{i} D_{j k}^{i}=0$ and $N_{i} D_{0 k}^{i}=0$. Therefore from (5) and (20) we get

$$
H_{\alpha}^{*}=\sqrt{\tau} H_{\alpha}
$$

If each path of a hypersurface $F^{n-1}$ with respect to the induced connection is also a path of the enveloping space $F^{n}$ then $F^{n-1}$ is called a hyperplane of the first kind. A hyperplane of the first kind is characterized by $H_{\alpha}=0$ [2]. Hence from (22) we have 
Theorem 4.1. If $b_{i}(x)$ be a vector field in a Finsler space $F^{n}$ satisfying (21) then a hypersurface $F^{n-1}$ is a hyperplane of the first kind if and only if the hypersurface $F^{* n-1}$ is a hyperplane of the first kind.

Next contracting (13) by $B_{\alpha}^{i} N^{* j} N^{* k}$ and paying attention to (19), $M_{\alpha}=$ $C_{i j k} B_{\alpha}^{i} N^{j} N^{k}, a_{i} N^{i}=0, h_{j k} N^{j} N^{k}=1$ and $h_{i j} B_{\alpha}^{i} N^{j}=0$, we get

$$
M_{\alpha}^{*}=M_{\alpha}-\frac{1}{2\left(e^{\sigma} L+\beta\right)} a_{i} B_{\alpha}^{i}
$$

From (5), (20), (21), (22) and (23) we have

$$
H_{\alpha \beta}^{*}=\sqrt{\tau}\left\{H_{\alpha \beta}-\frac{1}{2\left(e^{\sigma} L+\beta\right)} a_{i} B_{\alpha}^{i} H_{\beta}\right\}
$$

If each h-path of a hypersurface $F^{n-1}$ with respect to the induced connection is also h-path of the enveloping space $F^{n}$, then $F^{n-1}$ is called a hyperplane of the second kind. A hyperplane of the second kind is characterized by $H_{\alpha \beta}=0$ [2]. Since $H_{\alpha \beta}=0$ implies that $H_{\alpha}=0$ from (22) and (24) we have the following:

Theorem 4.2. If $b_{i}(x)$ be a vector field in a Finsler space $F^{n}$ satisfying (21) then a hypersurface $F^{n-1}$ is a hyperplane of the second kind if and only if the hypersurface $F^{* n-1}$ is a hyperplane of the second kind.

Finally contracting (13) by $B_{\alpha}^{i} B_{\beta}^{j} N^{* k}$ and paying attention to (6), (19), $a_{i} N^{i}=0$ and $h_{i j} B_{\alpha}^{i} N^{j}=0$, we have

$$
M_{\alpha \beta}^{*}=\sqrt{\tau} M_{\alpha \beta}
$$

If the unit normal vector of $F^{n-1}$ is parallel along each curve of $F^{n-1}$ then $F^{n-1}$ is called a hyperplane of the third kind. A hyperplane of the third kind is characterized by $H_{\alpha \beta}=0, M_{\alpha \beta}=0$ [2]. From (24) and (25) we have

Theorem 4.3. If $b_{i}(x)$ be a vector field in a Finsler space $F^{n}$ satisfying (21) then a hypersurface $F^{n-1}$ is a hyperplane of the third kind if and only if the hypersurface $F^{* n-1}$ is a hyperplane of the third kind.

\section{References}

[1] M. Kitayama, Finslerian hypersurface and metric transformation, Tensor, N.S., 60 (1998), 171-178.

[2] M. Matsumoto, The induced and intrinsic connections of a hypersurface and Finslerian projective geometry, J. Math. Kyoto Univ., 25 (1985), 107144. 
[3] C. Shibata, On invariant tensors of $\beta$-change of Finsler metric, J. Math. Kyoto Univ., 24 (1984), 163-188.

[4] M.S. Knebelman, Conformal geometry of generalized metric spaces, Proc. Nat. Acad. Sci. USA, 15 (1929), 33-41, 376-379.

[5] M. Hashiguchi, On conformal transformation of Finsler metric, J. Math. Kyoto Univ., 16 (1976), 25-50.

[6] H. Izumi, Conformal transformations of Finsler spaces I, Tensor, N.S., 31 (1977), 33-41.

[7] H. Izumi, Conformal transformations of Finsler spaces II, Tensor, N.S., 33 (1980), 337-359.

[8] M. Kitayama, Geometry of Transformations of Finsler Metrics, Hokkaido University of Education, Kushiro Campus., Japan (2000).

[9] S. Kikuchi, On theory of subspace, Tensor, N.S., 2 (1952), 67-79.

[10] H.S. Shukla, Arunima Mishra, On Finsler space with Randers conformal change: Main scalar, geodesic and scalar curvature, International J. Math. Combin., 3 (2012), 20-29.

[11] A. Moor, Finslerraume von identischer torsion, Acta Sci. Math., 34 (1973), 279-288.

[12] B.N. Prasad, B.K. Tripathi, Finslerian Hypersurfaces and Kropina change of a Finsler metric, Journal of Tensor Society of India, 23 (2005), 49-58. 